\title{
GORDURA CORPORAL E EFICIÊNCIA REPRODUTIVA EM ÉGUAS DOADORAS DE EMBRIÃO MANGALARGA MARCHADOR
}

\author{
Body fat and reproductive efficiency of Mangalarga Marchador embryo donor mares
}

\author{
Paula Gomes Rodrigues ${ }^{1}$, Camila de Moraes Raymundo², José Camisão de Souza², \\ Maria Cláudia Martins Guerra Miranda², Adalgiza Souza Carneiro de Rezende ${ }^{3}$
}

\begin{abstract}
RESUMO
Foi comparado o julgamento do escore de condição corporal (ECC) com a espessura de gordura subcutânea (EGS) e sua relação com a eficiência reprodutiva de éguas Mangalarga Marchador doadoras de embrião. O experimento foi conduzido com 56 éguas doadoras entre os meses de agosto/08 e abril/09. O ECC foi maior em éguas mais velhas e aumentou com os dias de permanência no experimento. Houve correlação positiva entre peso e tempo de experimento. O peso não foi associado à idade, porém aumentou ao longo do experimento. A EGS na região da cauda e entre a $12^{\mathrm{a}}$ e $13^{\mathrm{a}}$ costelas aumentou com a idade e diminuiu com os dias de permanência no experimento. Houve correlação positiva entre EGS e ECC para a cauda e costela. O diâmetro do folículo dominante aumentou ao longo do ciclo estral, e foi menor em éguas com ECC abaixo de 6,5. O diâmetro folicular não foi influenciado pela idade. O diâmetro médio do folículo ovulatório foi $38,43 \pm 3,09 \mathrm{~mm}$. A recuperação embrionária não foi afetada pela idade ou ECC, mas foi menor $(41 \%)$ eméguas que ovularam folículos menores que $40 \mathrm{~mm}$ quando comparados às que ovularam folículos maiores que $40 \mathrm{~mm}$ (59\%). A idade da doadora não foi associada à taxa de gestação dos embriões nas receptoras. A gordura corporal afetou a eficiência reprodutiva das éguas, além disso, a ultrassonografia parece ser essencial para a avaliação precisa e correta do escore de condição corporal.
\end{abstract}

Termos para indexação: Balanço energético, energia, equino, reprodução, transferência de embriões

\begin{abstract}
Body condition scoring (BCS) was compared to an ultrasound subcutaneous fat thickness (SFT) determination in relation to the reproductive efficiency of Mangalarga Marchador embryo donor mares. Fifty six barren mares were studied throughout the 2008/ 2009 breeding season (August through April) and the experiment was designed in a completely randomized scheme. BCS was higher in older mares and increased with time in the experiment, and there was a positive interaction between weight and time. Weight was not associated with age, but increased throughout the experiment. Tail and rib BCS increased with age, and decreased with time. There was a positive correlation between BCS and tail and rib SFT. The diameter of the dominant follicle increased during the estrous cycle and was smaller for mares with BCS below 6.5. Age did not influence the dominant follicle diameter. The mean ovulatory follicle diameter was $38.43 \pm 3.09$. Embryo recovery was not affected by age or BCS but was lower $(41 \%, \mathrm{P}=0.003)$ in mares that ovulated follicles smaller than $40 \mathrm{~mm}$ compared to mares ovulating follicles greater than $40 \mathrm{~mm}(59 \%)$. Donor age was not associated with recipient pregnancy rates. Body fat affected the reproductive efficiency in mares, and seems that ultra-sonography may be essential for correct and reliable body condition scoring.
\end{abstract}

Index termes: Embryo transfer, energy, energy balance, equine, reproduction

(Recebido em 8 de setembro de 2009 e aprovado em 29 de abril de 2011)

\section{INTRODUÇÃO}

O escore de condição corporal (ECC) é um método simples que não demanda equipamentos e estima a gordura corporal e, consequentemente, a quantidade de energia armazenada no corpo do animal (HENNEKE et al., 1983). Com treinamento adequado esse método permite alta repetibilidade entre observadores diversos. Fatores como a intensidade e frequência de trabalho, problemas parasitários e dentários, disponibilidade de água e manejo nutricional podem afetar o ECC.
Henneke et al. (1983) desenvolveram uma escala de ECC que varia de 1 até $9(1=$ animal excessivamente magro e 9 = animal excessivamente obeso) baseada na observação da aparência do animal e palpação da cobertura de gordura. Segundo os autores, esse método, quando devidamente aplicado, não é influenciado pelo tamanho, conformação, perímetro torácico, altura ou estágio fisiológico dos animais, pois encontraram correlação positiva entre ECC e porcentagem de gordura corporal, confirmando a hipótese de que esse sistema considera o animal como um todo e não apenas medidas individuais.

\footnotetext{
1Universidade Federal de Lavras/UFLA - Departamento de Zootecnia/DZO - Cx. P. 3037 - 37200-000 - Lavras, MG - paulagrodrigues@hotmail.com 2Universidade Federal de Lavras/UFLA - Departamento de Zootecnia/DZO - Lavras, MG

${ }^{3}$ Universidade Federal de Minas Gerais/UFMG - Departamento de Medicina Veterinária/DMV - Belo Horizonte, MG
} 
O ECC das éguas pode influenciar diversos componentes da eficiência reprodutiva, como taxa de concepção, intervalo de partos, duração da gestação, número de ciclos, duração do ciclo estral e perdas embrionárias (HENNEKE et al., 1983, HENNEKE et al., 1984; HINES et al., 1987; GASTAL et al., 2004).

Segundo o NRC (2007), o ECC 5 (moderado) é aquele considerado ideal para a obtenção da máxima eficiência reprodutiva das éguas. Henneke et al. (1984) observaram que éguas que entraram na estação de monta com ECC moderado apresentaram taxa de concepção mais alta quando comparadas com éguas que entraram na estação de monta mais magras, as quais apresentaram maior intervalo de partos e maior número de ciclos por concepção.

O conhecimento da relação entre gordura corporal e eficiência reprodutiva da égua doadora de embriões durante fases estratégicas de sua fisiologia, permite o estabelecimento de um plano nutricional adequado às suas condições corporais, otimizando seu aproveitamento reprodutivo e tornando o custo benefício o mais vantajoso possível aos criadores

O objetivo foi correlacionar o escore de condição corporal e a espessura de gordura subcutânea com a eficiência reprodutiva de éguas doadoras Mangalarga Marchador.

\section{MATERIAL E MÉTODOS}

O experimento foi conduzido no haras El Far, situado no Município de Lavras/MG. A coleta de dados ocorreu entre os meses de agosto/08 e abril/09. Foram utilizadas 56 éguas doadoras da raça Mangalarga Marchador, com faixa etária entre 4 e 22 anos, todas em condições normais de reprodução. As éguas foram mantidas em pasto de tanzânia (Panicum maximum cv Tanzânia) com sal mineral e água a vontade. Foram ainda submetidas a treinamento e condicionamento físico que consistia de aquecimento e marcha durante 30 ou 40 minutos em dias alternados.

O ECC foi avaliado mensalmente por meio da adaptação da técnica de Henneke et al. (1983) com acréscimo de 0,5 entre cada valor na escala de ECC. Foram criadas classes de ECC: classe $1=$ ECC $<6,5(n=355$ observações); classe $2=\mathrm{ECC} \geq 6,5$ e $<7,0$ ( $\mathrm{n}=263$ observações); classe $3=$ ECC $\geq 7,0$ e $\leq 7,5$ ( $n=549$ observações); classe $4=$ ECC > 7,5 ( $n=343$ observações). Para reduzir a subjetividade, o ECC utilizado foi obtido a partir da média de dois avaliadores. O peso foi estimado mensalmente com fita própria de pesagem para equinos (Ouro Fino ${ }^{\circledR}$, Cravinhos, SP).

A mensuração mensal da EGS foi feita com ultrassom Aloka SSD 500V com transdutor linear de 3,5 MHz. Dois pontos, ambos do lado direito do corpo do animal, foram utilizados para a determinação da EGS: cerca de $5 \mathrm{~cm}$ lateralmente à coluna vertebral entre $\mathrm{a} 12^{\mathrm{a}} \mathrm{e} 13^{\mathrm{a}}$ costelas, e $5 \mathrm{~cm}$ lateralmente à coluna vertebral e $7,0 \mathrm{~cm}$ cranialmente à inserção caudal (GENTRY et al., 2004). As imagens foram apresentadas no modo-B (Figura 1), gravadas em computador (programa Play TV USB 2.0, Prolink Computer Inc., Califórnia, EUA, 2000) e mensuradas pelo programa Image Pro-Plus 4.5 (Media Cybernetics, Silver Spring, EUA, 2002).

A evolução do diâmetro $(\mathrm{mm})$ do folículo dominante ovulatório foi acompanhada diariamente com ultrassom Aloka SSD 500V com transdutor linear de 5,0 $\mathrm{MHz}$, no modo B. As éguas foram inseminadas no dia da ovulação com sêmen fresco diluído em Botu-semen ${ }^{\circledR}$ (Biotech, Botucatu, SP) e a concentração espermática variou entre 800 milhões a 1 bilhão de espermatozóides/ $\mathrm{mL}$. A transferência do embrião foi realizada 8 dias após a ovulação da doadora, pelo método transcervical (não cirúrgico). Foi utilizado Ringer lactato (Glicolabor, Ribeirão

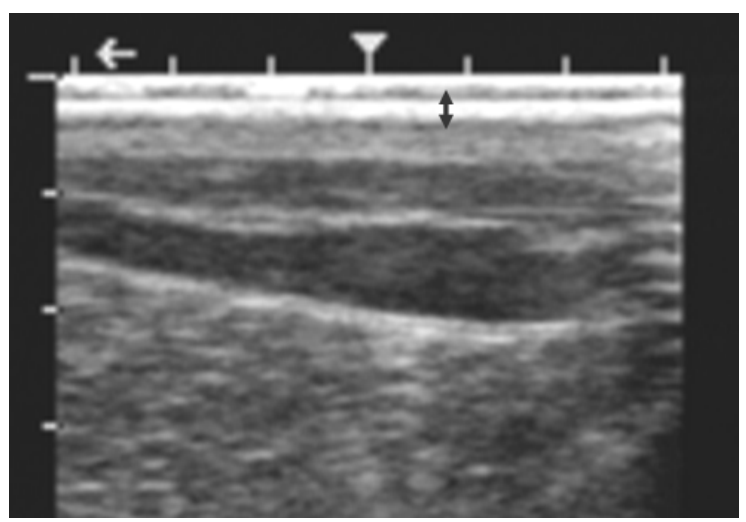

Figura 1 - Imagem de ultrassonografia com indicação da espessura da gordura subcutânea. 
Preto, SP) para a lavagem, cerca de 3 litros ou até obtenção do embrião.

Foi utilizado para todas as análises o pacote estatístico Statistical Analysis Systems, SAS ${ }^{\circledR}$ (1998). O delineamento estatístico foi inteiramente ao acaso. As variáveis medidas ao longo do tempo foram analisadas pelo procedimento Mixed, sendo égua dentro de tratamento testado como erro. A estrutura de covariância adotada em cada caso foi aquela com maior valor de Akaike. Todas as variáveis dependentes foram testadas quanto à normalidade pelo procedimento Univariate (SAS) que usa o teste de Kilmorov-Smirnov. As médias foram comparadas por contrastes ortogonais.

O efeito do ECC e idade sobre o diâmetro do folículo dominante foi analisado por análise de variância, assim como a análise dos efeitos fixos da idade e do tempo sobre o peso e o ECC. O modelo considerou o peso e o escore inicial, respectivamente, como covariáveis. Foram criadas 6 classes referentes ao tempo de permanência no experimento: classe $1=0-30$ dias $(n=96$ observações); classe $2=31-60$ dias $(n=53$ observações); classe $3=61$ 90 dias ( $\mathrm{n}=43$ observações $)$; classe $4=91-120$ dias $(\mathrm{n}=29$ observações); classe $5=121$-150 dias ( $\mathrm{n}=31$ observações) e classe $6=$ acima de 151 dias $(n=35$ observações $)$.

As éguas foram alojadas em classes de idades: classe 1 = idade $<7$ anos ( $\mathrm{n}=17$ éguas, com 81 observações); classe $2=$ idade $\geq 7$ e $\leq 13$ anos ( $n=25$ éguas, com 133 observações); classe $3=$ idade $>13$ anos ( $n=14$ éguas, com 73 observações) (BALL et al., 1987).

Os efeitos da idade e do tempo sobre a EGS foram avaliados pelo procedimento misto. Foi feita análise de correlação e teste de Spearman entre a EGS e o ECC. Análises de regressão foram realizadas entre ECC e peso, e entre o diâmetro do folículo dominante ovulatório ao longo dos dias do ciclo estral.

A relação entre taxa de recuperação embrionária e idade; idade e taxa de gestação dos embriões nas receptoras; ECC e taxa de recuperação embrionária; diâmetro do folículo dominante ovulatório e taxa de recuperação embrionária foram analisados pelo Qui Quadrado. Considerando a taxa de recuperação embrionária, como o número de embriões recuperados, em relação ao número de coletas por doadora, o nível de significância adotado foi de $5 \%$.

A mesma doadora pode ter produzido mais de um embrião ao longo do experimento e a mesma receptora pode ter recebido mais de um embrião, caso tenha perdido o embrião transplantado anteriormente. A taxa de gestação dos embriões das doadoras aos 30 dias nas éguas receptoras foi calculada por meio do número de éguas que tiveram o embrião transplantado em relação ao número de éguas gestantes aos 30 dias. Para a taxa de gestação aos 60 dias foi calculado, o número de éguas prenhes aos 30 dias em relação àquelas que mantiveram a gestação aos 60 dias. E para os 90 dias foi avaliado o número de éguas gestantes aos 60 dias em relação ao número de éguas que mantiveram a gestação aos 90 dias.

\section{RESULTADOSE DISCUSSÃO}

O peso das éguas não variou dentro das classes de idade $(\mathrm{P}<0,05)$, contudo aumentou ao longo dos dias de permanência no experimento $(\mathrm{P}<0,05)$, assim como o ECC $(\mathrm{P}<0,05)$. Tanto o peso quanto o ECC foram menores no primeiro mês de permanência no experimento, em seguida, aumentaram aos 31 dias e se mantiveram constantes até os 90 dias, e apresentaram seus maiores valores a partir de 91 dias de permanência no experimento até seu término, sem variações.

O ECC foi menor $(\mathrm{P}<0,05)$ em éguas com idade inferior a 6 anos em relação às éguas com idade entre 6 e 13 e acima de 13 anos (respectivamente $\mathrm{ECC}=6,8 ; 7,1$ e 7,12), que não diferiram entre si. O menor ECC encontrado nas éguas mais jovens pode ter ocorrido com base nos coeficientes de crescimento dos três principais tecidos que, por ordem crescente, são: tecido ósseo, muscular e adiposo (FRAPE, 2008). Como o ECC é definido como a quantidade de gordura (HENNEKE et al., 1983), cuja deposição ainda é incompleta em éguas jovens, pois, conforme a raça, o desenvolvimento corporal termina somente entre os 4 e 6 anos (MEYER, 1995), isso provavelmente resultou no menor ECC em relação aos animais mais velhos.

Houve relação linear positiva entre o peso e ECC $(\mathrm{P}<0,05)$, ou seja, o ECC aumentou com o ganho de peso das éguas. Resultados semelhantes foram obtidos por Carrol e Huntington (1988) e Lawrence et al. (1992).

AEGS foi maior em éguas mais velhas (Tabela 1), tanto na região da cauda como entre a $12^{\mathrm{a}}$ e $13^{\mathrm{a}}$ costelas $(\mathrm{P}<0,05)$. Éguas da classe de idade 1 apresentaram menor EGS do que aquelas das classes de idade 2 e 3, que não diferiram entre si, tanto na região da cauda, quanto entre a $12^{\mathrm{a}}$ e $13^{\mathrm{a}}$ costelas. Percentualmente, o aumento da EGS na região da cauda foi de $7,86 \%$ e o aumento entre a $12^{\mathrm{a}}$ e $13^{\mathrm{a}}$ costelas foi de $11,6 \%$.

É importante salientar que esse aumento se refere somente às regiões medidas por ultrassom nos pontos pré-determinados. Esse percentual em ganho de gordura corporal deve ser considerado para o corpo do animal como um todo, exemplificando, dessa forma, a grande quantidade de gordura que o animal ganha ao longo da vida. Provavelmente, éguas mais velhas tendem a acumular mais 
gordura devido, em razão da taxa metabólica que ocorre quando o equino atinge cerca de 15 anos de idade (MEYER, 1995; ANDRIGUETTO et al., 1983) o que corrobora os resultados observados.

Ao longo dos dias do experimento, houve diminuição da EGS, tanto da região da cauda $(\mathrm{P}<0,05)$ quanto entre a $12^{\mathrm{a}}$ e $13^{\mathrm{a}}$ costelas $(\mathrm{P}<0,05)$ (Tabela 2). Aparentemente, a diminuição da gordura na região da cauda foi percentualmente maior do que aquela observada entre a $12^{\mathrm{a}}$ e $13^{\mathrm{a}}$ costelas, respectivamente $17,2 \%$ e $12,9 \%$. Resultados semelhantes foram obtidos por Gentry et al. (2004), que observaram que o armazenamento de gordura na região da cauda é maior em relação a qualquer outra parte do corpo equino e, quanto mais cranialmente a esta região, menor é a deposição de gordura.

É provável que a atividade física tenha provocado queima de gordura e desenvolvimento de massa muscular, o que explicaria o aumento de peso e ECC ao mesmo tempo em que ocorreu diminuição de gordura. Westervelt et al. (1976) obtiveram resultados similares com cavalos de pólo, detectando diminuição da EGS na garupa e cernelha 60 e 90 dias após o início de exercícios físicos intensos. Dessa forma, para animais que realizam atividade física moderada ou intensa, em condições semelhantes às desse experimento, o ECC proposto por Henneke et al. (1983) pode não ser indicativo adequado da cobertura de gordura corporal. De maneira semelhante, Suagee et al. (2008) também concluíram que esse método de avaliação de ECC para cavalos de corrida não foram suficientes para a determinação correta da gordura corporal desses animais atletas.

Houve correlação positiva entre a EGS na região da cauda $(\mathrm{P}<0,05)$ e entre a $12^{\mathrm{a}}$ e $13^{\mathrm{a}}$ costelas $(\mathrm{P}<0,05)$ com o ECC. A EGS aumentou com o ganho de ECC das éguas. Lawrence et al. (1992) e Gentry et al. (2004) obtiveram resultados semelhantes.

$\mathrm{O}$ diâmetro folicular foi semelhante para todas as classes de idade $(\mathrm{P}>0,05)$, cerca de $34,21 \pm 0,37 \mathrm{~mm}$. Winter et al. (2007) e Ginther et al. (2009) encontraram resultados similares. O diâmetro do folículo dominante ovulatório apresentou relação linear positiva com a aproximação do dia de ocorrência da ovulação (Figura 2).

O ECC afetou o diâmetro folicular (Tabela 3). As classes deECC 1 e 4 apresentaram menor diâmetro folicular,

Tabela 1 - Efeito da idade sobre a espessura $(\mathrm{cm})$ de gordura subcutânea na região da cauda (EGSCA) e entre a $12^{\mathrm{a}}$ e $13^{\text {a }}$ costelas (EGSCO). Valores são médias ${ }^{1}$ dos quadrados mínimos (lsmeans \pm erro padrão).

\begin{tabular}{ccccc}
\hline & \multicolumn{3}{c}{ Classes de idade $^{2}$} & \multirow{2}{*}{ Probabilidade } \\
\cline { 2 - 4 } & 1 & 2 & 3 & $\mathrm{P}<0,05$ \\
EGSCA $^{1}$ & $1,40^{\mathrm{a}} \pm 0,04$ & $1,53^{\mathrm{b}} \pm 0,03$ & $1,51^{\mathrm{b}} \pm 0,04$ & $\mathrm{P}<0,05$ \\
EGSCO $^{1}$ & $1,38^{\mathrm{a}} \pm 0,04$ & $1,51^{\mathrm{b}} \pm 0,03$ & $1,54^{\mathrm{b}} \pm 0,05$ & \\
\hline Número de observações & 73 & 132 & 81 & \\
\hline
\end{tabular}

${ }^{1}$ Diferença entre médias foram testadas por contrastes ortogonais. ${ }^{2}$ Classes de idade, sendo: 1 = abaixo dos 6 anos (n=17 éguas); $2=$ maior ou igual a 6 anos e menor e igual a 13 anos ( $\mathrm{n}=25$ éguas); 3 = maior que 13 anos ( $\mathrm{n}=14$ éguas).

Tabela 2 - Efeito dos dias de permanência no experimento sobre a espessura $(\mathrm{cm})$ de gordura subcutânea na região da cauda (EGSCA) e entre a $12^{\mathrm{a}}$ e $13^{\mathrm{a}}$ costelas (EGSCO). Valores são médias ${ }^{1}$ dos quadrados mínimos (lsmeans \pm erro padrão).

\begin{tabular}{ccccccc}
\hline & \multicolumn{6}{c}{ Classes de tempo $^{4}$} \\
\cline { 2 - 7 } & 1 & 2 & 3 & 4 & 5 & 6 \\
\hline EGSCA $^{2}$ & $1,57^{\mathrm{a}}$ & $1,51^{\mathrm{ab}}$ & $1,54^{\mathrm{ab}}$ & $1,50^{\mathrm{bc}}$ & $1,43^{\mathrm{c}}$ & $1,30^{\mathrm{d}}$ \\
& $\pm 0,03$ & $\pm 0,03$ & $\pm 0,03$ & $\pm 0,03$ & $\pm 0,04$ & $\pm 0,04$ \\
EGSCO $^{3}$ & $1,55^{\mathrm{a}}$ & $1,53^{\mathrm{a}}$ & $1,53^{\mathrm{a}}$ & $1,49^{\mathrm{ab}}$ & $1,42^{\mathrm{bc}}$ & $1,35^{\mathrm{c}}$ \\
& $\pm 0,03$ & $\pm 0,03$ & $\pm 0,04$ & $\pm 0,04$ & $\pm 0,04$ & $\pm 0,05$ \\
Número de observações & 95 & 53 & 43 & 29 & 31 & 35 \\
\hline
\end{tabular}

${ }^{1}$ Diferença entre médias foram testadas por contrastes ortogonais. ${ }^{2}$ Probabilidade: $\mathrm{P}<0,05 .{ }^{3}$ Probabilidade: $\mathrm{P}<0,05 .{ }^{4} \mathrm{Classes}$ de tempo (dias de permanência no experimento): 1 - 0 a 30 dias; 2 - 31 a 60 dias; 3 - 61 a 90 dias; $4-91$ a 120 dias; 5 - 121 a 150 dias; 6 - acima de 151 dias. 
referentes, respectivamente, às éguas com ECC abaixo de 6,5 e acima de 7,5. Já, o maior diâmetro folicular foi observado nas éguas das classes 2 e 3 , respectivamente éguas de ECC entre 6,5 e 7,5. Gastal et al. (2000), Godoi et al. (2002) e Gastal et al. (2004) também observaram correlação positiva entre o diâmetro máximo dos folículos ovulatórios e o ECC.

Não houve efeito $(\mathrm{P}>0,05)$ do ECC sobre a taxa de recuperação embrionária (Tabela 4). Entretanto, o maior diâmetro do folículo dominante foi associado com a melhora da taxa de recuperação embrionária $(\mathrm{P}<0,05)$.

Éguas que ovularam um folículo com diâmetro inferior a $40 \mathrm{~mm}$ tiveram menor $(\mathrm{P}<0,05)$ taxa de embriões recuperados no lavado $(41,06 \%)$ do que éguas com folículos de diâmetro igual ou superior a $40 \mathrm{~mm}(58,94 \%)$. De acordo com Ginther (2000) e Hafez e Hafez (2004), o maior diâmetro folicular está intimamente relacionado com células da granulosa mais ativas e, consequentemente, com melhor qualidade do ovócito e do embrião subsequente que será formado.

A idade das doadoras não afetou a taxa de recuperação embrionária $(\mathrm{P}>0,05)$ e também não foi capaz de exercer efeito $(\mathrm{P}>0,05)$ sobre a taxa de gestação dos embriões nas éguas receptoras aos 30 dias de prenhez (Tabela 5).

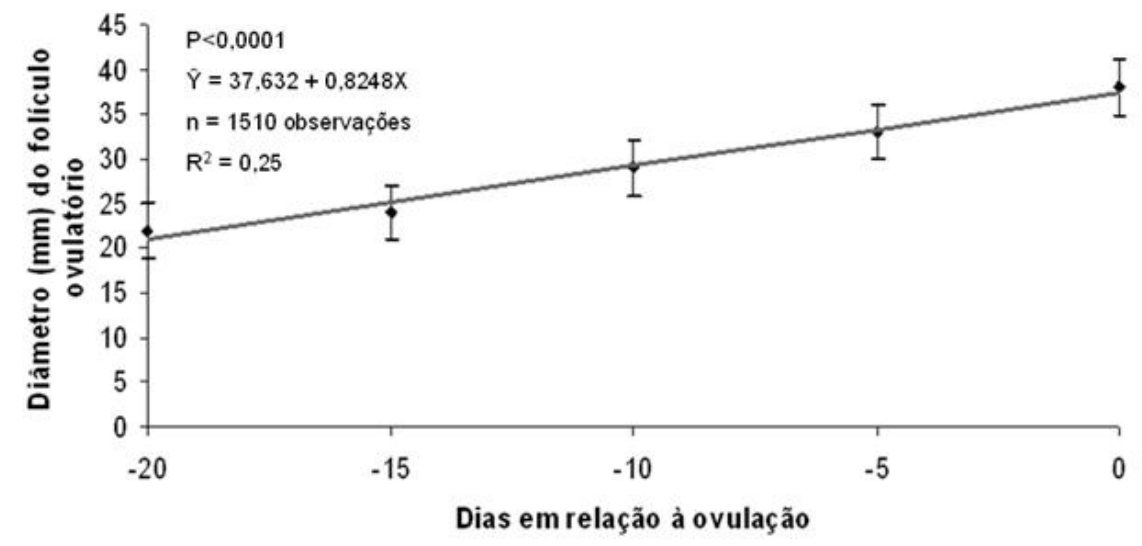

Figura 2 - Diâmetro (mm) médio do folículo dominante ovulatório ao longo dos dias do ciclo estral.

Tabela 3 - Efeito do escore de condição corporal sobre o diâmetro (mm) médio do folículo dominante ovulatório. Valores são médias ${ }^{1}$ dos quadrados mínimos (lsmeans \pm erro padrão).

\begin{tabular}{|c|c|c|c|c|c|}
\hline & \multicolumn{4}{|c|}{ Classes de Escore $^{2}$} & \multirow{2}{*}{ Probab } \\
\hline & 1 & 2 & 3 & 4 & \\
\hline Diâmetro folicular & $33,80^{\mathrm{a}} \pm 0,31$ & $34,95^{\mathrm{c}} \pm 0,36$ & $34,40^{b c} \pm 0,25$ & $34,17^{\mathrm{ab}} \pm 0,31$ & $\mathrm{P}<0,05$ \\
\hline Número de observações & 355 & 263 & 549 & 343 & \\
\hline
\end{tabular}

${ }^{1}$ Diferença entre médias foram testadas por contrastes ortogonais. ${ }^{2}$ Classes de escore corporal, sendo: $1=\mathrm{ECC}<6,5 ; 2=6,5 \geq$ $\mathrm{ECC}<7,0 ; 3=7,0 \geq \mathrm{ECC} \leq 7,5 ; 4=\mathrm{ECC}>7,5$.

Tabela 4 - Efeito do diâmetro $(\mathrm{mm})$ médio do folículo dominante ovulatório sobre a taxa de recuperação embrionária (\%). Valores são médias ${ }^{1}$ dos quadrados mínimos (lsmeans \pm erro padrão).

\begin{tabular}{cccc}
\hline & \multicolumn{2}{c}{ Diâmetro do folículo dominante ovulatório } & \multirow{2}{*}{ Probabilidade } \\
\cline { 2 - 3 } & $<40 \mathrm{~mm}$ & $\geq 40 \mathrm{~mm}$ & $\mathrm{P}<0,05$ \\
Taxa de recuperação (\%) & 41,06 & 58,94 & \\
Número de observações & 124 & 178 & \\
\hline
\end{tabular}

${ }^{1}$ Diferença entre médias foram testadas por contrastes ortogonais.

Ciênc. agrotec., Lavras, v. 35, n. 5, p. 1002 -1008, set./out., 2011 
Tabela 5 - Idade (anos) das doadoras em relação à taxa de recuperação embrionária (\%) e taxa de gestação (\%) dos embriões das doadoras aos 30 dias nas receptoras. Valores são médias ${ }^{1}$ dos quadrados mínimos (lsmeans \pm erro padrão).

\begin{tabular}{ccc}
\hline Classes de idade $^{2}$ & Taxa de recuperação embrionária (\%) & Taxa de gestação $(\%)$ aos 30 dias \\
\hline 1 & $52,33(29 / 55)^{3}$ & $91,67(18 / 20)^{4}$ \\
2 & $57,24(108 / 188)$ & $100,00(77 / 77)$ \\
3 & $47,89(37 / 77)$ & $90,91(20 / 22)$ \\
\hline Probabilidade & $\mathrm{P}>0,05$ & $\mathrm{P}>0,05$
\end{tabular}

${ }^{1}$ Diferença entre médias foram testadas por contrastes ortogonais. ${ }^{2}$ Classes de idade, sendo: $1=$ abaixo dos 6 anos $(\mathrm{n}=17$ éguas); $2=$ maior ou igual a 6 anos e menor e igual a 13 anos ( $\mathrm{n}=25$ éguas); 3 = maior que 13 anos (n=14 éguas). ${ }^{3}$ Número de embriões recuperados em relação ao número de lavados realizados. ${ }^{4}$ Número de receptoras gestantes em relação ao número de receptoras de embriões.

Houve tendência $(\mathrm{P}<0,10)$ de menor taxa de gestação de embriões transplantados provenientes de doadoras com idade inferior a 7 anos e maior que 13 anos, e também de menor taxa de recuperação embrionária em éguas com mais de 13 anos. Em contraste, Ginther et al. (2004) observaram efeitos negativos da idade sobre a taxa de recuperação embrionária, resultados que podem estar associados ao número reduzido de folículos e à qualidade inferior dos oócitos frequentemente observados em éguas mais velhas.

\section{CONCLUSÃO}

O escore de condição corporal influenciou o diâmetro do folículo dominante ovulatório das éguas e, indiretamente, afetou também a proporção de embriões recuperados, pois folículos de maior diâmetro resultaram em taxas de recuperação embrionária mais elevadas. Dessa forma, o escore de condição corporal pode ser indicado como ferramenta de manejo para o monitoramento de éguas doadoras e receptoras com o objetivo de melhorar a eficiência reprodutiva.

\section{AGRADECIMENTOS}

À Fundação de Amparo a Pesquisa do Estado de Minas Gerais, pelo apoio financeiro. Ao Haras El Far por permitir a realização deste trabalho.

\section{REFERÊNCIAS BIBLIOGRÁFICAS}

ANDRIGUETTO, J. M. et al. Nutrição animal: as bases e os fundamentos da nutrição animal. São Paulo: Nobel, 1983. v. 2, 425 p.

BALL, B. A. et al. Survival of day 4 embryos transferred from the oviduct of normal and sub-fertile donor mares to the uterus of normal recipient mares. Theriogenology, v. 27, n. 1, January, p. 208-215, 1987.
CARROLL, C. L.; HUNTINGTON, P. J. Body condition scoring and weight estimation of horses. Equine Veterinary Journal, Cambridge, v. 20, n. 1, p. 41-45, Apr. 1988.

FRAPE, D. Nutrição \& alimentação de equinos. 3. ed. São Paulo: Rocca, 2008. 602 p.

GASTAL, M. O.et al. Body condition influences diameter of the ovulatory follicle in mares. Biology of Reproduction, Champaign, v. 62, n. 2, p. 222-234, Nov. 2000.

GASTAL, M. O. et al. Relationships between body condition and follicle development in mares. Animal Reproduction Science, Amsterdan, v. 1, n. 1, p. 115-121, Oct/Dec., 2004.

GENTRY, L. R. et al. The relationship between body condition score and ultrasonic fat measurements in mares oh high versus low body condition. Journal of Equine Veterinary Science, Wildomar, v. 24, n. 5, p. 198203, May 2004.

GINTHER, O. J. Selection of the dominant follicle in cattle and horses. Animal Reproduction Science, Amsterdam, v. 60, p. 61-79, July 2000. Supplement.

GINTHER, O. J. et al. Seasonal influence on equine dynamics. Animal Reproduction Science, Amsterdam, v. 1, n. 1, p. 31-44, Oct./Dec. 2004.

GINTHER, O. J. et al. Age-related dynamics of follicles and hormones during an induced ovulatory follicular wave in mares. Theriogenology, Stoneham, v. 71, n. 5, p. 780-788, Mar. 2009. 
GODOI, D. B.; GASTAL, E. L.; GASTAL, M. O. A comparative study of follicular dynamics between lactating and non-lactating mares: effect of the body condition. Theriogenology, Stoneham, v. 58, n. 2, August, p. 553-556, 2002.

HAFEZ, E. S. E.; HAFEZ, B. Reprodução animal. 7. ed. São Paulo: Manole, 2004. 513 p.

HENNEKE, D. G; POTTER, G. D.; KREIDER, J. L. Body condition during pregnancy and lactation and reproductive efficiency in mares. Theriogenology, Stoneham, v. 21, n. 6, p. 897-909, June 1984.

HENNEKE, D. R. et al. Relationship between body condition score, physical measurements and body fat percentage in mares. Equine Veterinary Journal, Cambridge, v. 15, n. 4, November, p. 371-372, 1983.

HINES, K. K. et al. G. Relationship between body condition and levels of serum luteinizing hormone in postpartum mares. Theriogenology, Stoneham, v. 28, n. 6, p. 815-825, Dec. 1987.

LAWRENCE, L. M. et al. Changes in body weight and condition of gestanting mares. Journal of Equine Veterinary Science, Wildomar, v. 12, n. 6, p. 355-358, Nov./Dec. 1992.
MEDIA CYBERNETICS. Image Pro-Plus: aplication notes. Silver Spring, 2002.50 p.

MEYER, H. Alimentação de cavalos. 2. ed. São Paulo: Varela, 1995. 303 p.

NATIONALRESEARCHCOUNCIL(NRC). Nutrients Requeriments of Horses. Washington: National Academy of Sciences, 2007. 341 p.

PROLINK COMPUTER. Play TV USB 2.0: user's guide. California, 2000. 30p.

STATISTICALANALYSYS SYSTEMS - SAS. User's guide: version 6. Cary, NC: SAS Institute, 1998. 1686 p.

SUAGEE, J. K. et al. Effects of diet and weight gain on body condition coring in Throughbred geldings.

Journal of Equine Veterinary Science, Wildomar, v. 28, n. 3, p. 156-166, Mar. 2008.

WESTERVELT, R. G. et al. Estimating fatness in horses and ponies. Journal of Animal Science, Champaign, v. 43, n. 4, p. 781-785, Oct. 1976.

WINTER, G. H. Z. et al. Gestational Length and First Postpartum Ovulation of Criollo Mares on a Stud Farm in Southern Brazil. Journal of Equine Veterinary Science, Wildomar, v. 27, p. 531-534, 2007. 